\title{
Dynamic Surface and Interfacial Tension of AFFF and Fluorine-free Class B Foam Solutions
}

\author{
BOGDAN Z. DLUGOGORSKI, SATIT PHIYANALINMAT, and \\ ERIC M. KENNEDY \\ Process Safety and Environment Protection Research Group \\ School of Engineering, The University of Newcastle \\ Callaghan, NSW 2308, AUSTRALIA
}

\begin{abstract}
This paper investigates the dynamic surface tension of aqueous film forming (AFFF) and fluorine-free (FF) foam solutions by pendant drop tensiometry. Additional experiments are conducted to measure the interfacial tension between the foam solutions and two hydrocarbon liquids, $n$-heptane and diesel oil. The pendant drop technique yields both surface and interfacial tension at time scales ranging from seconds to hours, being limited at the lower end by the time necessary to form a droplet, and at the upper end by the evaporation of the droplet's material. The results indicate that the surface tension of AFFF formulations, diluted at the design concentration, rapidly reaches its equilibrium value. However, the approach to equilibrium is slowed down by additives, such as xanthan gum, present in the alcohol tolerant concentrates (ATC-AFFF). FF solutions show a slower approach to static values than AFFF formulations. At the design dilution, and at room temperature, these static values are in the order of $27 \mathrm{mN} \mathrm{m}^{-1}$, as compared to $16 \mathrm{mN} \mathrm{m}^{-1}$ for AFFF solutions. The measurements of the interfacial tension indicate rapid attainment of equilibrium for both AFFF and FF formulations, with the static values of around 2 and $0.9-2.5 \mathrm{mN} \mathrm{m}^{-1}$, for AFFF and FF, respectively. With the surface tension of $n$-heptane and diesel oil of 20.1 and $28.3 \mathrm{mN} \mathrm{m}^{-1}$, the spreading coefficient of $\mathrm{FF}$ formulations for the present systems varies between -9.4 and $0.6 \mathrm{mN} \mathrm{m}^{-1}$. These results indicate that film formation does not play a role during fire suppression by FF foams.
\end{abstract}

KEYWORDS: class B foams, AFFF, fluorine-free foams, ATC-AFFF, AR-AFFF, RF3, RF6, dynamic surface tension, dynamic interfacial tension, spreading coefficient

\section{INTRODUCTION}

The introduction of fluoro surfactants into formulations of fire fighting foams was perhaps one of the most important innovations in the past century in fire protection chemistry for the rapid control and extinguishment of flammable liquid fires. The resulting product was named Light Water ${ }^{\mathrm{TM}}$ Aqueous Film Forming Foam (AFFF). AFFF foam technology has become known for its key performance characteristics: rapid knockdown and extinguishment; effectiveness in extinguishing spill and storage tank fires; long shelf life; self-healing; ability to secure fuel spills and control post-fire fuel evaporation; compatibility with dry chemicals; ease of foaming with conventional nozzles; and capacity to be applied through standard sprinkler heads.

The fluorosurfactants have a unique structure that involves a combination of perfluorinated, hydrocarbon and head group segments [1,2]. The result is a surface active agent that has a water soluble (hydrophilic) end and a perfluorinated end that is non-water soluble (hydrophobic) end. In addition to repelling water, the hydrophobic part of the fluorinated surfactant also repels hydrocarbon liquids and oils; that is, it possesses the 
lipophobic properties. In effect, the perfluorinated end positions itself in the air within a three-phase air-water-oil system, facilitating the formation of thin ( $20 \mu \mathrm{m}$ in thickness) films that spread rapidly on hydrocarbon surfaces [3]. Unfortunately, concerns have been raised about the health effects of the degradation products of fluorosurfactants in the environment. The organic portions of fluorosurfactant molecules appear to biodegrade quite rapidly, leaving the fluorinated segment of the chain as inert and nonbiodegradable. Such perfluorinated segments do not occur naturally in the environment [1]. It is now well accepted that certain fluorosurfactant chemistries, especially those based on perfluorooctyl sulphonate (PFOS) and perfluorooctanoic acid (PFOA) derivatives decompose in the environment to products that are bioaccumulative and toxic $[4,5]$. For this reason, a number of fluorine-free (FF, this term was introduced by Willson [6]) foams have been developed, with two of these formulations approaching the performance of AFFF or FFFP (film forming fluoroprotein foams). One emulates fluoroprotein foam (manufactured by Angus Fire Armour, but not known to be commercially available), whereas the second imitates AFFF/FFFP performance (manufactured by 3M Company) [7].

From this perspective, the aim of this paper is to examine the performance characteristics of the new FF formulations. Specifically, we pose two focussing questions: (i) Are FF foams capable of forming thin films, and does the film formation play a role in the extinguishment of fires by these foams? (ii) What is the effect of concentration and temperature on the evolution of surface and interfacial tension for FF foams, and how do these properties compare with those of AFFF formulations? To address the second question, we intend to perform measurements with two AFFF formulations, namely FC3002 and FC600, with the latter formulation incorporating xanthan gum and other additives to make it an alcohol tolerant concentrate (ATC). We also restrict ourselves to perform measurements of the dynamic tension at time scale longer than $1 \mathrm{~s}$, as we suspect that FF solutions would display a slower approach to equilibrium than AFFF formulations and wish to generate the data that would be useful for modelling the effect of dynamic surface and interfacial tension on flow, drainage and coarsening of FF foams. The characteristic time for film spreading of AFFF films is in the order of 0.1 to $1 \mathrm{~s}$ [3], whereas fire fighting foam formulations exhibit a characteristic time for foam flow (as opposed to film flow), drainage and coarsening of between 10 and $600 \mathrm{~s}$ [2]. Until present, the dynamic surface and interfacial tension has not been included in modelling of these phenomena in the context of fire fighting foams [8-11]. Consequently, we selected the pendant drop technique [12] rather than the maximum bubble pressure tensiometry [13] to perform the surface-tension measurements.

This article is structured as follows: The next chapter describes the materials used and the experimental methodology. The subsequent chapter, covering the discussion of the experimental measurements, is divided into two sections, one on the dynamic surface tension and the other on the dynamic interfacial surface tension. The latter also discusses the spreading coefficient for FF formulations. The major findings of this paper are summarised in conclusions.

\section{MATERIALS AND METHODS}

Samples of two fluorosurfactant-based (AFFF (FC3002), ATC-AFFF (FC600)) and two fluorine-free (RF3 and RF6) concentrates were obtained from local suppliers in Australia. Table 1 summarises the approximate composition of the concentrates. With reference to the table, the major hydrocarbon surfactants used in the formulations are diethanolamine 
lauryl sulphate and cocamidopropyl hydroxy sultaine, whereas the major fluorocarbon surfactants include amphoteric fluoroalkylamide derivatives and perfluoroalkyl sulphonate salts. The foam concentrates also incorporate small amounts of biocides, corrosion inhibitors, preservatives, retardants, and other agents, as reviewed recently by Schaefer [7].

Table 1. Chemical composition of foam concentrates used in the present study.

\begin{tabular}{|l|c|c|c|c|}
\cline { 2 - 5 } \multicolumn{1}{c|}{} & $\begin{array}{c}\text { AFFF } \\
\text { FC3002, \% }\end{array}$ & $\begin{array}{c}\text { ATC-AFFF } \\
\text { FC600, \% }\end{array}$ & $\begin{array}{c}\text { RF3 } \\
\text { \% }\end{array}$ & $\begin{array}{c}\text { RF6 } \\
\text { \% }\end{array}$ \\
\hline Water & $50-60$ & $77-85$ & $<75$ & $<80$ \\
\hline Diethyl glycol butyl ether & $30-40$ & $10-15$ & $5-10$ & $5-10$ \\
\hline Hydrocarbon surfactants & $1-6$ & $1-5$ & $<5$ & $<10$ \\
\hline Fluorocarbon surfactants & $1-7$ & $1-6.5$ & - & - \\
\hline Thickeners, carbonised sugars & - & $1-5$ & $<17$ & $<12$ \\
\hline
\end{tabular}

The concentrates were diluted with distilled water to the required concentration of between 0.01 and $3 \%$ of concentrate content, with solutions assuming a light amber colour. The solutions were prepared immediately prior to experiments and never stored for longer than one day. GC grade heptane (Riedel de-Haën, Germany) and automotive grade diesel oil (Shell, Australia) were used without modification for the measurements of the dynamic interfacial tension.

The viscosity of AFFF concentrate appeared similar to that of water, whereas the concentrates of RF3, RF6 and ATC-AFFF were substantially more viscous as a consequence of the presence of the xanthan gum thickener. Xanthan gum prevents settling of other components in the concentrates, extending the concentrates' shell life. This effect occurs as a result of yield stress exhibited by solutions of xanthan gum. Also, the solutions of xanthan gum possess an exceptionally small dependence of viscosity on temperature, minimising the effects of temperature on pressure drop during pumping and induction of foam concentrates, and enhancing protection against freezing; the latter effect is also facilitated by diethylene glycol butyl ether. Furthermore, xanthan gum stabilises the interface between air and foam solution, and make that interface less mobile, slowing down foam drainage. This effect is very similar to that exhibited by protein foams [9]. Finally, xanthan gum enhances the suppression of fires of polar liquids. However, it is presently unknown whether xanthan gum modifies the dynamic behaviour of the surface and interfacial tension of solutions of fire fighting foams.

The dynamic surface and interfacial tension was measured using a pendant drop tensiometer, model OCA20, manufactured by Data Physics (Fig. 1). At long times, the technique also yields measurements of static tension, provided that the effect of evaporation of droplet material remains negligible. The Data Physics instrument is designed to acquire dynamic tension values of between $10^{-2}$ and $2 \times 10^{3} \mathrm{mN} \mathrm{m}^{-1}$. A dispenser unit, controlled by a PC based software, manipulates the injection of liquid into the needle $(1.65 \mathrm{~mm}$ in outside diameter) to form pendant droplets. This operation requires approximately one second to complete, and hence the instrument cannot provide surface age measurements at time scale of less than $1.5 \mathrm{~s}$. In the present experiments the droplets were $10-15 \mu \mathrm{L}$ in volume for the dilute solutions of the surfactants, down to 
around $5 \mu \mathrm{L}$ for more concentrated solutions. For the measurement of the interfacial tension, the fluid cell was filled with $n$-heptane or diesel oil. Distilled water served to provide measurements of the surface tension after each experiment involving foam solutions to verify the cleanliness of the needle tip.

As surfactant molecules diffuse from the bulk and adsorb at the interface, the surface tension decreases and a droplet elongates. A CCD camera (Fig. 1), with a resolution of $768 \times 576$ pixels, captures this elongation in real time. The camera supports the field of view that can vary between $1.75 \times 1.4$ to $11.7 \times 9 \mathrm{~mm}$, with the distortion of less than $0.05 \%$. A clear and contrasted image of a droplet constitutes a prerequisite for the subsequent analysis. Once a droplet forms, images are taken and recorded on the computer's hard drive for subsequent processing.

\section{RESULTS AND DISCUSSION}

\section{Dynamic Surface Tension}

Figure 2 compares the dynamic surface tension of AFFF (FC3002), ATC-AFFF (FC600) solutions with RF3 and RF6 foam solutions, at $25^{\circ} \mathrm{C}$ and two levels of dilution, namely 0.1 and $3 \%$. It is immediately evident in the figure that the 3\% solution of FC3002 reaches its static surface tension of $16.3 \mathrm{mN} \mathrm{m}^{-1}$ within $2 \mathrm{~s}$; i.e., in a time shorter than allowed by the limitation of the instrument; see Table 2 for the equilibrium data. In the case of ATC-AFFF, slightly more time (around $5 \mathrm{~s}$ ) is required for the system to approach the equilibrium surface tension; although, the magnitude of this tension $\left(16.2 \mathrm{mN} \mathrm{m}^{-1}\right)$ is essentially the same as for FC3002. A similar equilibrium value for FC3002 and FC600 may imply that both of the solutions contain the same type of fluorosurfactants. This comment follows from the observation that, in mixtures of fluoro and hydrocarbon surfactants with high enough concentration of fluorosurfactants, the equilibrium surface tension reflects the fluorosurfactant rather than hydrocarbon surfactant present [1].

We propose that the small difference in time required to reach the equilibrium between AFFF and ATC-AFFF is a consequence of xanthan gum present in the ATC-AFFF formulation. Xanthan molecules appear to provide an additional resistance to the diffusion of fluorosurfactant molecules to the interface, which manifests itself in a slower approach to the equilibrium surface tension.

RF3 and RF6, both diluted by mixing 3 parts of foam concentrate with 97 parts of distilled water, yield the equilibrium surface tension of 26.8 and $26.7 \mathrm{mN} \mathrm{m}^{-1}$, respectively. The approach to equilibrium for RF3 and RF6 solutions takes approximately 5 s, similarly as for FC600. Both RF3 and RF6 concentrates contain xanthan gum [6]. This further corroborates our earlier conclusion of the effect of xanthan gum on slowing down the diffusion of surfactants to the interface. From a practical perspective, the presence of xanthan gum reduces the rate of evolution of the dynamic surface tension, and hence it retards the spreading of thin films of AFFF solutions. 


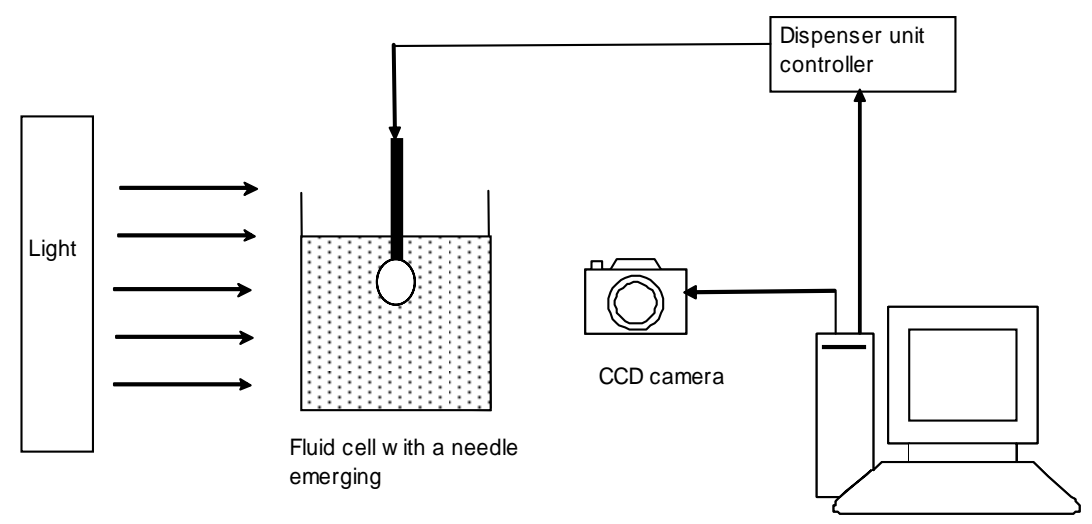

Fig. 1. Schematic diagram of the experimental apparatus.

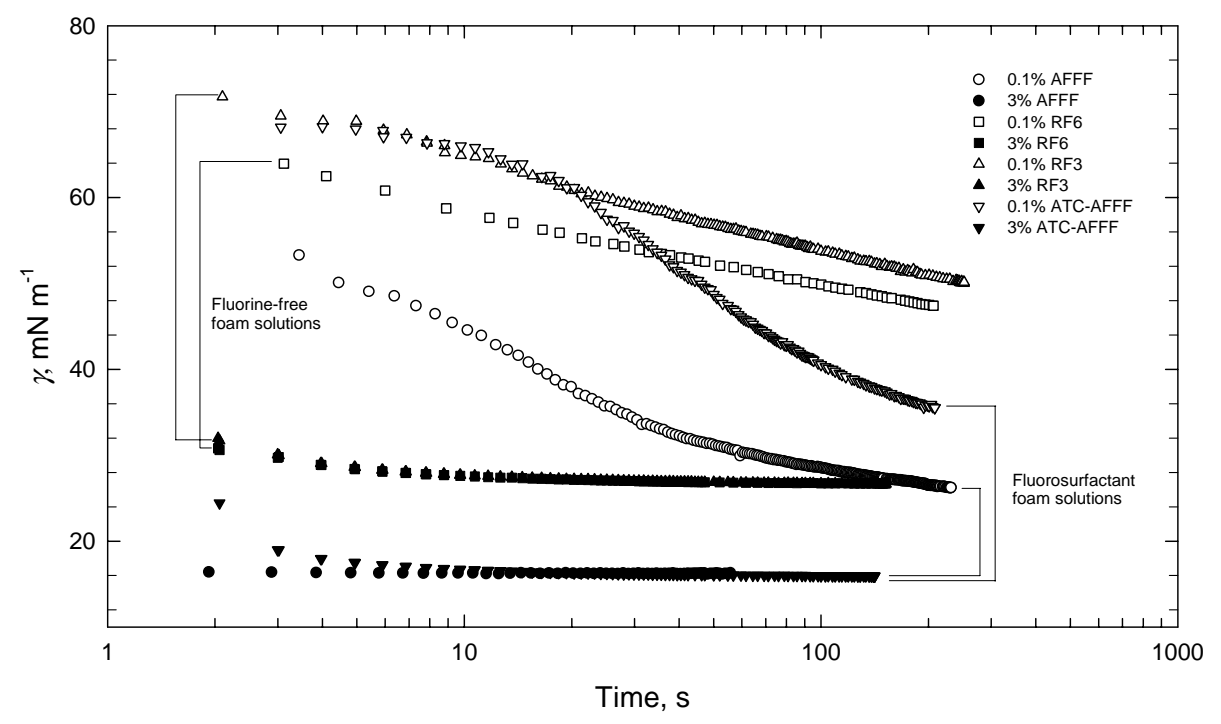

Fig. 2. Comparison of the evolution of dynamic surface tension for the four AFFF and FF foam solutions considered in this study. The measurements were performed at $25^{\circ} \mathrm{C}$. The symbol $\gamma$ in the ordinate's label stands for the dynamic surface tension.

Figure 2 also compares the variation of the dynamic surface tension of the foam solutions diluted to $0.1 \%$ content of the concentrate. At this dilution level, only FC3002 approaches its equilibrium surface tension value of $26.3 \mathrm{mN} \mathrm{m}^{-1}$, within the first $200 \mathrm{~s}$ of the experiment. It is estimated that the $0.1 \%$ solution of FC600 would take an order of magnitude longer to approach its static tension. On the other hand, at $0.1 \%$ dilution, RF solutions tend to decrease their surface tension with time at a much slower pace. It is known that the rate of the approach to equilibrium depends on whether the surfactant concentration is above or below the critical micelle concentration (CMC) [14]. The CMC for AFFF formulations is around $0.2-0.3 \%$, as concluded by plotting the measurements of the third row of Table 2 in terms of the static surface tension versus the logarithm of concentration. Thus, the very rapid approach to static values for $3 \%$ solution is not 
surprising, as this concentration is 10 times higher than CMC. ATC-AFFF approaches the equilibrium more slowly than AFFF. One may hypothesise that this is an effect of the interaction of xanthan gum with the surfactants present in the system. Finally, at $0.1 \%$ dilution, RF3 as well as RF6 appear to lie below their CMC, with RF6 approaching the equilibrium faster than RF3. The reason for this behaviour is unclear, as the RF3 concentrate would be expected to have higher surfactant content and lower xanthan gum content, with both of these factors promoting rather than retarding the approach to equilibrium.

Table 2. Equilibrium surface tension of FC3002, FC600, RF3 and RF6 foams at $25^{\circ} \mathrm{C}$, at various levels of dilution.

\begin{tabular}{|l|c|c|c|c|c|c|c|}
\cline { 2 - 9 } \multicolumn{1}{c|}{} & \multicolumn{7}{c|}{ Equilibrium surface tension, $\mathbf{~ m ~ m}^{-1}$} \\
\cline { 2 - 9 } \multicolumn{1}{c|}{} & $3 \%$ & $1 \%$ & $0.3 \%$ & $0.2 \%$ & $0.1 \%$ & $0.03 \%$ & $0.01 \%$ \\
\hline AFFF, FC3002 & 16.3 & 16.8 & 17.6 & 20.9 & 26.3 & 49.1 & 56.3 \\
\hline ATC-AFFF, FC600 & 16.2 & - & - & - & - & - & - \\
\hline RF3 & 26.8 & - & - & - & - & - & - \\
\hline RF6 & 26.7 & 27.3 & - & - & - & - & - \\
\hline
\end{tabular}

Note that the curves of the dynamic surface tension of AFFF and ATC-AFFF are similar to each other. Likewise, the curves of the dynamic surface tension for RF3 and RF6 closely resemble each other. For this reason, in the subsequent discussion we consider FC3002 and RF6 as proxies for fluorosurfactant and FF foam classes, respectively, and investigate only these two formulations in more detail. Specifically, Figs. 3 and 4 compare the effect of the concentration of surfactants on the dynamic surface tension of FC3002 and RF6 solutions. In Fig. 3 note the two replicates for $0.06 \%$ dilution. The comparison of the two replicates indicates that, the measurements presented in this article are reproducible to within $4 \%$.

Three effects become immediately evident when one compares the experimental measurements presented in Figs. 3 and 4. Firstly, the presence of even small amounts of fluorosurfactants tends to decrease the surface tension substantially more than the equivalent amount of hydrocarbon surfactants; e.g., compare curves in Figs. 3 and 4 for $0.01 \%$. This obviously indicates that fluorosurfactants are much more surface active than hydrocarbon surfactants, in spite of their similar adsorption effectiveness at the interface [15]. Secondly, both for RF6 and AFFF, the curves reflecting the dilution between 0.2 and $3 \%$ appear to converge to approximately the same equilibrium values; just above $16 \mathrm{mN} \mathrm{m}^{-1}$ for AFFF and in excess of $26 \mathrm{mN} \mathrm{m}^{-1}$ for RF6. This again suggests that micelles start to form at concentration of around $0.2-0.3 \%$. The values of the static surface tension will be similar but not exactly the same as the dilution varies between CMC and 3\%; Table 2 illustrates this point further for AFFF formulation between 0.3 and $3 \%$. The reason for this phenomena lies in the activity of the surfactants in the bulk which increases with increasing surfactant concentration, having small but noticeable effect on the equilibrium surface tension [15]. Thirdly, even above CMC, but at lower surfactant concentration, it takes longer for the molecules to diffuse to the interface and to orient their hydrophobic chains into the air. Macroscopically, this behaviour leads to slower evolution of the dynamic surface tension. Practically, this means that, AFFF 
formulations, for which one desires a very rapid attainment of the equilibrium on the time scale of less than a second, must not be diluted to below their design concentration (usually 3\%). Otherwise, the velocity of film spreading will decrease and the foam performance will start to deteriorate accordingly. These considerations are of less significance for RF6 formulations that do not employ film spreading as the primary suppression mechanism. Finally, we remark that mixtures of fluoro and hydrocarbon surfactants tend to display dynamic surface tension that is lower than that of the individual components [13]. Whether this mechanism operates in the present systems would need to be confirmed with experiments on single surfactants.

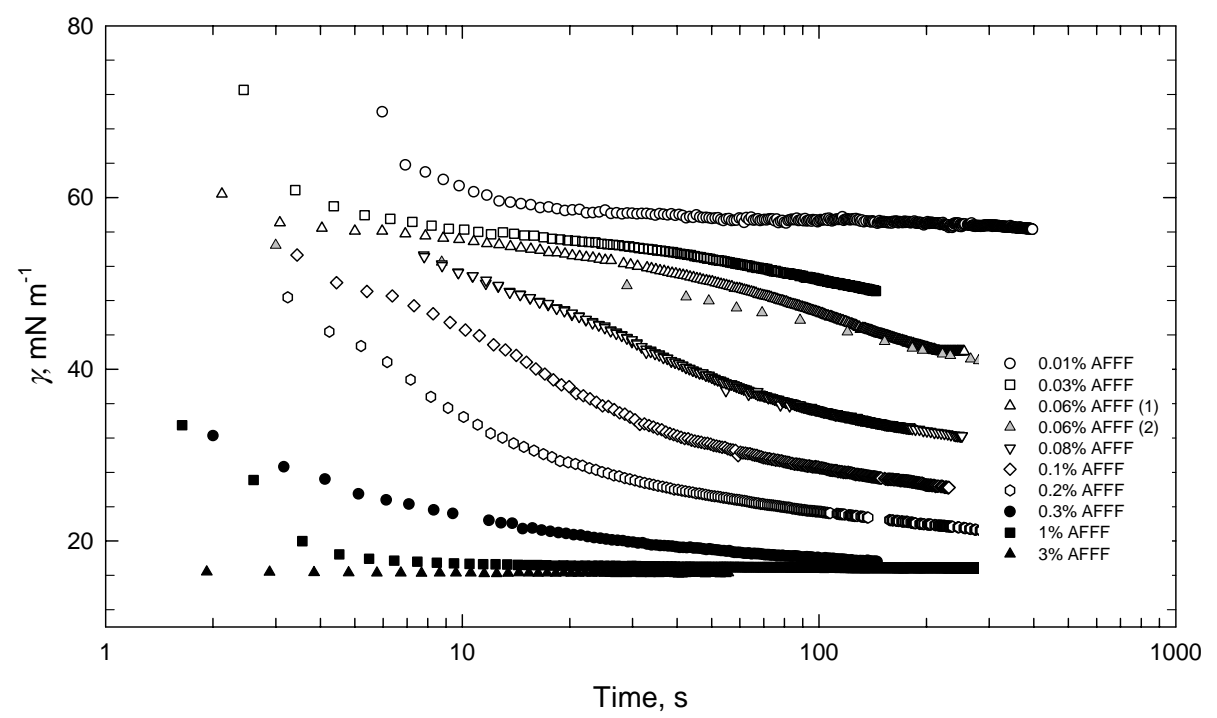

Fig. 3. The effect of dilution of AFFF concentrate on the dynamic surface tension between 0.01 to $3 \%$, at $25^{\circ} \mathrm{C}$.

We have also performed a preliminary investigation of the effect of temperature on the dynamic surface tension of FC3002 and RF6 solutions (0.1 and 3\%), carrying out experiments at $35^{\circ} \mathrm{C}$. For a $0.1 \%$ solution, the surface tension for RF6 decreases substantially at higher temperatures. At the same temperature, the behaviour of FC3002 was however more complex, with the dynamic surface tension at $35^{\circ} \mathrm{C}$ lying initially below that of $25^{\circ} \mathrm{C}$, with the crossover at around $10 \mathrm{~s}$. At $3 \%$, the measurements for AFFF at 25 and $35^{\circ} \mathrm{C}$ coincided, whereas for RF6 we observed faster approach to equilibrium and lower static value at $35^{\circ} \mathrm{C}$ than at $25^{\circ} \mathrm{C}$; 25.6 in comparison to $26.8 \mathrm{mN} \mathrm{m}^{-1}$ at $25^{\circ} \mathrm{C}$. These preliminary measurements are too fragmentary to conclude about the relative importance of diffusion and adsorption mechanisms and about the effect of temperature on the thermodynamic equilibrium. 


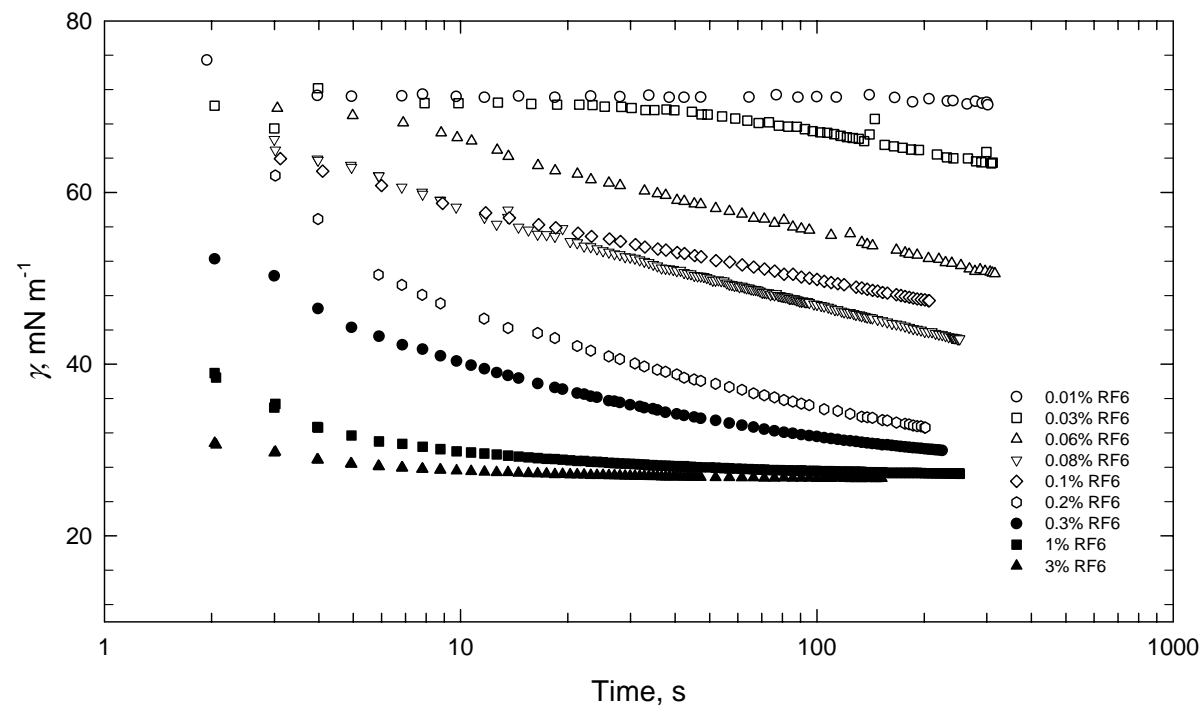

Fig. 4. The effect of dilution of RF6 concentrate on the dynamic surface tension between 0.01 to $3 \%$, at $25^{\circ} \mathrm{C}$.

\section{Dynamic Interfacial Tension}

To study the dynamic interfacial surface tension, we again selected AFFF and RF6 solutions at two concentration levels of 0.1 and $3 \%$ in contact with single ( $n$-heptane) and multicomponent (diesel oil) hydrocarbon fuels. The results are plotted in Figs. 5 and 6, with the equilibrium data for 3\% dilution presented in Table 3, both for 25 and $35^{\circ} \mathrm{C}$. Except for the 3\% AFFF/n-heptane, systems containing diesel oil tend to approach an equilibrium faster that those based on $n$-heptane. We explain this behaviour by recalling the multicomponent nature of diesel oil; i.e., diesel may incorporate components that are surface active and diffuse towards the interface contributing to the reduction in the interfacial surface tension.

The difference between the interfacial tension for heptane and diesel is more significant for RF6 than for AFFF formulations, especially at lower $(0.1 \%)$ content of the concentrates in the solution. We posit that the hydrophobic chains of the fluorosurfactants (which are also lipophobic) induce the formation of a structure that lies flat on the hydrocarbon side of the interface. This creates a barrier to the diffusion of more polar components (such as additives) of the diesel oil across the interface, and results in similar dynamic interfacial tension for $n$-heptane and diesel oil systems. The situation is however different in the case of RF6 formulation. The hydrocarbon chains are not only hydrophobic but also lipophilic, forming a perpendicular structure in the fuel at the interface. This structure is less coherent at lower concentrations of surfactants, allowing the diffusion of components of the diesel oil across the interface into the aqueous solution of RF6, engendering significantly lower dynamic interfacial tension in RF6/diesel oil than RF6/n-heptane systems. Schaefer et al. [16] have recently reported that AFFF formulations suppress vapours of hydrocarbon liquids more efficiently than FF foams. Although Schaefer et al. performed their experiments with $n$-hexane rather than diesel oil, their results indicate, consistent with the present measurements, that, non-fluorosurfactant formulations do not perform as well as AFFF for suppression of vapours of hydrocarbon liquids. 


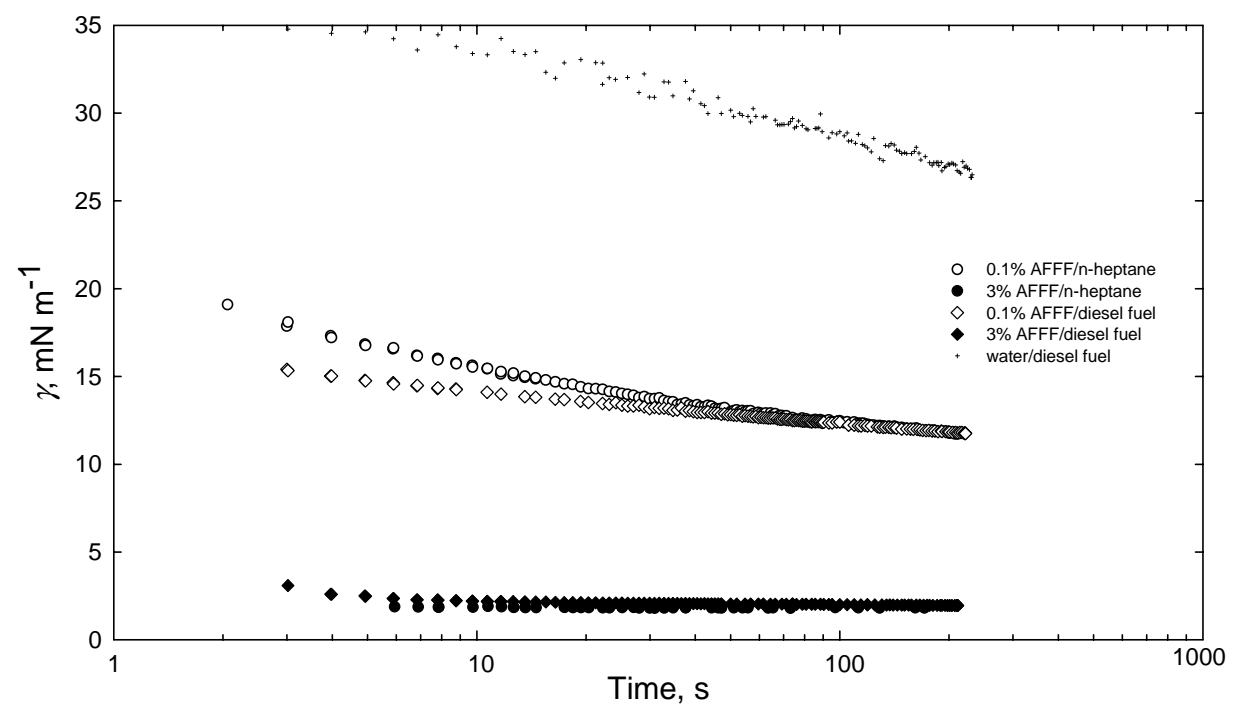

Fig. 5. The evolution of dynamic interfacial tension for AFFF ( 0.1 and $3 \%)$ in contact with $n$-heptane and diesel oil; all measurements are at $25^{\circ} \mathrm{C}$.

Similarly to the measurement of the effect of temperature on the surface tension of RF6 and FC3002 at 3\% concentration, we observed only a small effect of temperature on the interfacial surface tension, with the equilibrium values listed in Table 3. It is worth noting that, at this concentration, for 3 out of 4 systems studied in the article, the static interfacial tension remains constant or decreases with temperature. Only for RF6/nheptane, there is a noticeable increase from 2.5 to $3.2 \mathrm{mN} \mathrm{m}^{-1}$. At $0.1 \%$, RF6/n-heptane system showed a significant decrease in the dynamic surface tension at $35^{\circ} \mathrm{C}$, whereas for other systems we observed the characteristic crossover at around 8-20 s.

Table 3. Equilibrium interfacial tension of FC3002/n-heptane, FC3002/diesel, RF6/n-heptane and RF6/diesel, at 3\% concentrate content.

\begin{tabular}{|l|c|c|}
\cline { 2 - 3 } \multicolumn{1}{c|}{} & \multicolumn{2}{c|}{ Equilibrium interfacial tension, $\mathbf{~} \mathbf{~} \mathbf{~ m}^{\mathbf{- 1}}$} \\
\cline { 2 - 3 } \multicolumn{1}{c|}{} & $\mathbf{2 5}^{\mathbf{0}} \mathbf{C}$ & $\mathbf{3 5}^{\mathbf{0}} \mathbf{C}$ \\
\hline AFFF/n-heptane & 1.8 & 2.1 \\
\hline AFFF/diesel oil & 2.0 & 2.0 \\
\hline RF6/n-heptane & 2.5 & 3.2 \\
\hline RF6/diesel oil & 0.9 & 0.9 \\
\hline
\end{tabular}

The present measurements indicate that RF6 foams in contact with diesel oil may show slightly higher fuel pick-up than AFFF foams or RF6 foams in contact with $n$-heptane, as a consequence of the rather low values of the interfacial tension. Indeed, when aqueous solutions were poured into test probes containing hydrocarbons and then shaken, the emulsion formed in RF6/diesel oil system was the most stable. 


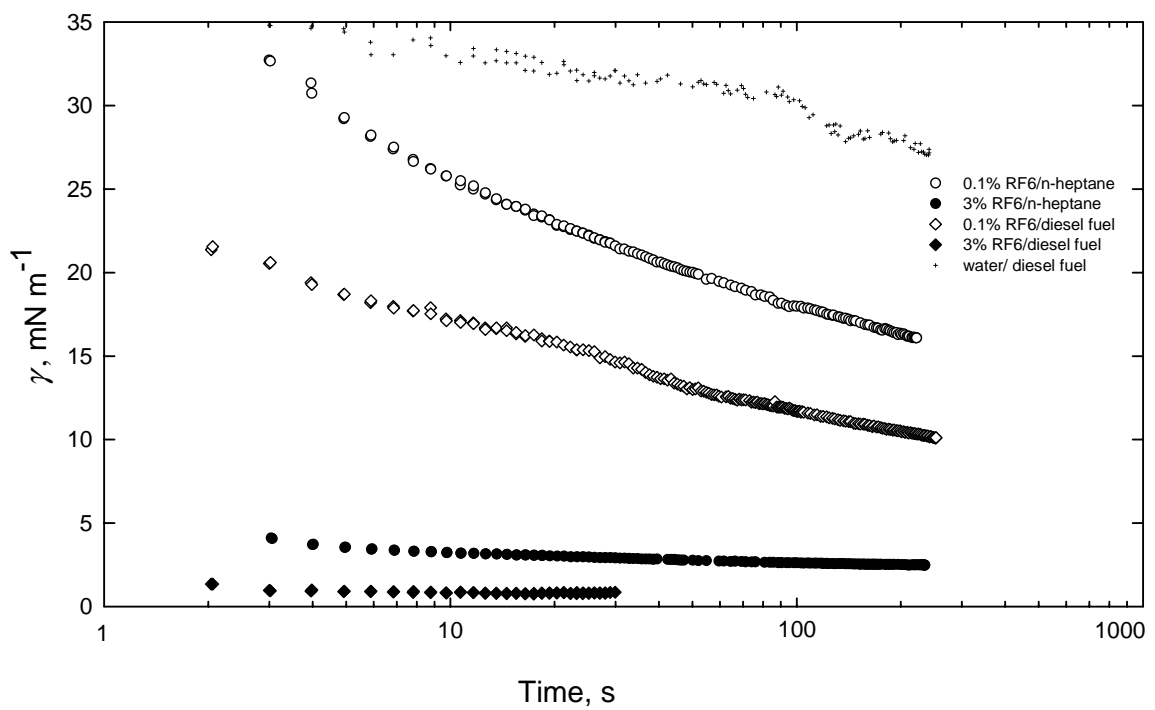

Fig. 6. The evolution of dynamic interfacial tension for RF6 (0.1 and 3\%) in contact with $n$-heptane and diesel oil; all measurements are at $25^{\circ} \mathrm{C}$.

The spreading of thin films on surfaces of hydrocarbon liquids is proportional to the dynamic spreading coefficient $S(t)$ [3] defined as

$S(t)=\gamma_{h c}(t)-\left[\gamma_{f}(t)+\gamma_{f / h c}(t)\right]$

where $\gamma_{h c}(t)$ denotes the dynamic surface tension of a hydrocarbon liquid, for single component liquids $\gamma_{h c}$ is constant, $\gamma_{f}(t)$ is the dynamic surface tension of a foam solution and $\gamma_{f h c}(t)$ stands for the dynamic interfacial tension between a foam solution and a hydrocarbon liquid. The spreading commences once a sufficient quantity of surfactant diffuses and adsorbs at the interface to prompt $S$ to exceed zero.

As measured in this study, the surface tension of $n$-heptane and diesel fuel is 20.1 and $28.3 \mathrm{mN} \mathrm{m}^{-1}$ at $25^{\circ} \mathrm{C}$, and 19.4 and $27.3 \mathrm{mN} \mathrm{m}^{-1}$ at $35^{\circ} \mathrm{C}$; these values are similar to those published in the literature [17]. By replacing these values in Eq. 1 together with the measurements plotted in Fig. 3 and interpolating the data in Fig. 5, we conclude that AFFF solutions would not spread on $n$-heptane unless the concentrate level exceeds its critical micelle concentration. As a matter of fact, the static spreading coefficient $(t \rightarrow \infty)$ for AFFF on heptane is only $1.1 \mathrm{mN} \mathrm{m}^{-1}$, indicating that spreading may start only several seconds after the application of AFFF solution. This means that, to realise its fire suppression effectiveness, AFFF solutions must be used at their design concentration. Furthermore, this observation leads us to remark that it is the effective suppression of fuel vapours by AFFF films rather than film spreading, per se, that is the most important feature of fluorosurfactant based formulations.

For diesel oil, the static spreading coefficient is around $10 \mathrm{mN} \mathrm{m}^{-1}$, pointing to rapid spreading of thin films. This is one of the reasons that diesel oil fires are much easier to extinguish by AFFF foams than $n$-heptane fires. (The other is the lower vapour pressure of diesel oil, and hence better sealing of diesel oil vapours by thin AFFF films.) Only on 
diesel oil, FF formulations display a small positive static spreading coefficient. In other systems, this coefficient is as low as $-9.4 \mathrm{mN} \mathrm{m}^{-1}$. This suggests that, thin FF films would not spread on most hydrocarbon liquids, and therefore would not contribute to the observed extinguishment. However, RF6 formulation has passed the stringent ICAO level $\mathrm{B}$ test protocol [16]. This means that FF foams must mitigate fires by mechanisms other than film spreading. The FF foams themselves (rather than FF films) move rapidly on the surface of hydrocarbon liquids. They have good heat resistance that allows for fast fire securement. If the foam blanket is broken, the bubbles flow back to fill the foam discontinuity; i.e., FF foams are capable of repairing structural damage in foam coverage.

\section{CONCLUSIONS}

By reporting the dynamic surface and interfacial tension, this contribution has demonstrated that FF solutions do not form thin films on surfaces of hydrocarbon liquids. Thus foams, produced by aerating FF solutions, must extinguish the fires by mechanisms other than film forming. Most likely the FF foams themselves spread rapidly on burning surfaces. Likewise, the FF foams themselves provide a sealing barrier to diffusion of flammable vapours. The dynamic tension of FF solutions shows slower approach to equilibrium than AFFF solutions; though, this is not of practical relevance for FF solutions, since film formation does not contribute to suppression of fires by FF foams.

Detailed analyses of the dynamic surface and interfacial tension indicate that AFFF solutions diluted to below their critical micelle concentration are unable to form spreading films, even at long times. Only, AFFF formulations diluted to their design concentration (3\%) can provide effective fire extinguishment. AFFF formulations diluted to between $\mathrm{CMC}$ and $3 \%$ are characterised by progressively shorter times necessary for the surface and interfacial tension to decrease to allow the spreading coefficient to become positive. It is proposed that the addition of xanthan gum slows the diffusion of the surfactants and their adsorption at the interface, a phenomenon that may affect the efficiency of fire extinguishment by the alcohol tolerant formulations.

It is also observed that the spreading coefficient of AFFF on non-aromatic hydrocarbon fuels is small, in the order of $1 \mathrm{mN} \mathrm{m}^{-1}$. This indicates that the suppression effectiveness of AFFF formulations against fires of non-aromatic hydrocarbons is a consequence of sealing properties of the fluorosurfactant films rather than their spreading.

\section{REFERENCES}

[1] Kissa, E., Fluorinated Surfactants and Repellents, Marcel Dekker Inc., New York, 2001.

[2] Dlugogorski, B.Z., Kennedy, E.M., Schaefer, T.H., and Vitali, J., "What Properties Matter in Fire-fighting Foams?” Proc. $2^{\text {nd }}$ NRIFD Symp., Tokyo, 2002, pp. 57-78.

[3] Jho, C., "Spreading of Aqueous Solutions of a Mixture of Fluoro- and Hydrocarbon Surfactants on Liquid Hydrocarbon Substrates," Journal of Colloid and Interface Science, 117, Iss. 1, pp. 139-148, (1987).

[4] Renner, R., "Growing Concern Over Perfluorinated Chemicals," Environmental Science and Technology, 35, pp. 154A-160A, (2001). 
[5] Moody, C.A., and Field, J.A., "Perfluorinated Surfactants and the Environmental Implications of Their Use in Fire Fighting Foams," Environmental Science and Technology, 34, pp. 3864-3870, (2000).

[6] Willson, M., “Are ‘Environment Friendly’ Foams Really Green?” International Fire Protection Magazine, (1), (2004), http://www.iffmag.com/articles/issue1/foam.htm.

[7] Schaefer, T.H., Aqueous Foaming Composition, PCT/US02/38937, WO 03/049813 A1, World Intellectual Property Organization, p. 22, 2003.

[8] Magrabi, S.A., Dlugogorski, B.Z., and Jameson, G.J., "Bubble Size Distribution and Coarsening of Aqueous Foams," Chemical Engineering Science, 54, pp. 4007-4032, (1999).

[9] Magrabi, S.A., Dlugogorski, B.Z., and Jameson, G.J., "A Comparative Study of Drainage Characteristics in AFFF and FFFP Compressed-air Fire-fighting Foams,” Fire Safety J, 37, pp. 21-52, (2002); see also AIChE Journal, 47, pp. 314-327, (2001).

[10] Persson, B., Lönnermark, A., Persson, H., Mulligan, D., Lancia, A., and Demichela, M., Foamspex: Large Scale Foam Application - Modelling of Foam Spread and Extinguishment, SP, Borås, 2001, p. 273.

[11] Lattimer, B., and Hanauska, C., "The Use of Small-scale Test Data to Characterize Some Aspects of Fire Fighting Foam for Suppression Modelling,” Fire Safety Journal, 38, pp. 117-146, (2003).

[12] Lin, S.Y., McKeigue, K., and Maldarelli, C., "Diffusion-controlled Surfactant Adsorption Studied by Pendant Drop Digitisation," AIChE Journal, 36, pp. 1785-1795, (1990).

[13] Hirt, D.E., Prud'homme, R.K., Miller, B. and Rebenfeld, L., "Dynamic Surface Tension of Hydrocarbon and Fluorocarbon Surfactant Solutions Using the Maximum Bubble Pressure Method," Colloids and Surfaces, 44, pp. 101-117, (1990).

[14] Klein, H.G., Meussdoerffer, J.N., Niederprm, H., and Wechsberg, M., "Tetraethylammonium Perfluorooctanesulfonate, a Versatile Fluorosurfactant with Many Uses,” Tenside Detergents, 15, Iss. 1, pp. 2-6, (1978).

[15] Adamson, A.W., and Gast, A.P., Physical Chemistry of Surfaces, John Wiley \& Sons, New York, 1997.

[16] Schaefer, T.H., Dlugogorski, B.Z., and Kennedy, E.M., "Class B Fire-fighting Foams - Performance Balanced with Environment,” Proc. Fire Safety at Sea, Melbourne, Australia, CD-ROM, pp. 1-18, 2004.

[17] Escobedo J. and G.A. Mansoori (1996) "Surface Tension Prediction for Pure Fluids” AIChE Journal 45, 1425-1433. 